\title{
PERSPECTIVA DO FARMACÊUTICO NO USO DA RITALINA POR ACADÊMICOS
}

\author{
PHARMACIST'S PERSPECTIVE ON THE USE OF RITALIN BY ACADEMICS
}

\author{
Alessandro Galoni Madriaga ${ }^{1}$ \\ Vicente Antonio de Senna Junior ${ }^{2}$
}

RESUMO: É notório que o uso da Ritalina por acadêmicos tem aumentado no decorrer dos anos, esse aumento se dá devido aos efeitos "benéficos" que a Ritalina pode proporcionar aos estudantes mesmo sabendo que o medicamento não é prescrito para essa finalidade. A Ritalina tem como princípio ativo o metilfenidato, este é classificado como psicoestimulante, utilizado para o tratamento de transtorno de déficit de atenção e hiperatividade (TDAH), em crianças e adultos, que consumido em doses corretas, pode auxiliar no tratamento, contribuindo para o desempenho de tarefas. Essa pesquisa tem como objetivo explorar sobre o uso da Ritalina e a automedicação por alunos do nível superior, os possíveis efeitos colaterais e as conseqüências do uso da Ritalina, ressaltando a necessidade de uma investigação maior sobre o uso da Ritalina por parte dos estudantes, principalmente a longo prazo.

Palavras-chave: Metilfenidato. Ritalina. TDAH. Automedicação e Conseqüências. Efeitos colaterais. Acadêmicos.

ABSTRACT: It is notorious that the use of methylphenidate by academics has increased over the years, this increase is due to the "beneficial" effects that Ritalin can provide to students despite the drug not having a prescription for this purpose. Ritalin's active ingredient is methylphenidate, which is classified as a psychostimulant, used for the treatment of attention deficit hyperactivity disorder (ADHD) in children and adults, which consumed in correct doses, can help in the treatment, contributing to the performance of tasks. This research aims to investigate the use of Ritalin and selfmedication by higher education students, the possible side effects and consequences of the use of Ritalin, highlighting the need for further investigation on the use of Ritalin by students, especially long-term.

Keywords: Methylphenidate. Ritalin. ADHD. Delf-medication and consequences. Side effects. Academic.

\footnotetext{
', Bacharel em Farmácia pela Faculdade Iguaçu- UNIG. E-mail: alessandrogmadriaga@gmail.com ${ }^{2}$ Orientador. Mestre em Saúde pública pela fundação Oswaldo Cruz -FIOCRUZ. Professor da universidade iguaçu - UNIG ( orientador).e-mail: sennajr@yahoo.com.br.
} 


\section{INTRODUÇÃO}

O Metilfenidato (MPH) foi sintetizado pela primeira vez em 1944, já em 1955, a companhia farmacêutica Ciba-geygy (precursora da Novartis) foi responsável por lançar o produto no mercado, utilizando o nome de Ritalina (Nunes et al., 2020). Ao iniciar os anos 6o a Ritalina começou a ser utilizada no tratamento do Transtorno de Deficit de Atenção e Hiperatividade (TDAH).

O Transtorno de Deficit de Atenção/Hiperatividade (TDAH) ou Transtorno Hipercinético é um distúrbio de comportamento em crianças e adolescentes. Cerca de 3\% das crianças sofrem deste transtorno, o que os torna incapazes de ficarem paradas e/ou se concentrar em tarefas por um determinado período de tempo. As crianças com esse transtorno podem ter dificuldades para aprender a fazer tarefas escolares. Elas podem freqüentemente se tornar difícil de lidar, tanto na escola quanto em casa.

No Brasil começou a ser comercializada por volta de 1988 , tornando-se o maior consumidor mundial de Ritalina (ITABORAHY, 2009). Além do tratamento do TDAH, o metilfenidato é utilizado também para o uso recreativo, para buscar mais para o lazer, uso estético, sugestão de utilidade para ajudar no emagrecimento, e o uso cognitivo, que busca ter uma maior ampliação, relacionadas às capacidades psíquicas (BRAZ et al.,2020).

Diante disso, o presente projeto de pesquisa tem como objeto de estudo a perspectiva do farmacêutico para com o uso indiscriminado de Ritalina por acadêmicos, ressaltando a importância da atenção farmacêutica no uso desse medicamento.

Primeiramente será realizado um diagnóstico sobre o uso da Ritalina com a prescrição médica e a indicação para seu uso, será abordado também seu uso de forma irracional, as conseqüências de seu uso apontando para os riscos, principalmente quando usado a longo prazo.

Espero que este trabalho possa enriquecer e levar a compreensão que o uso da Ritalina é prescrito para pacientes adultos e crianças portadoras do transtorno de déficit de atenção e hiperatividade (TDAH), desmitificando a cultura criada por acadêmicos que com o uso da Ritalina é possível manter um bom rendimento e aprendizado diante das exigências de 
produtividade que a vida acadêmica exige. Sendo assim, faz-se necessário divulgar o conhecimento sobre esse assunto, pois muitos desses usuários desconhecem os efeitos colaterais que o medicamento proporciona, e acabam não levando em consideração que se trata de um fármaco utilizado para tratamento de TDAH, sendo visto apenas como um grande aliado no aumento da produtividade acadêmica auxiliando para um melhor desempenho.

\section{OBJETIVOS}

\section{2.r. Objetivo Geral}

Investigar sobre o uso da Ritalina e automedicação por estudantes do nível superior, enfatizando a importância do farmacêutico no uso racional de medicamentos, fornecendo informações necessárias acerca do medicamento a fim de prevenir possíveis problemas relacionados a farmacoterapia

\subsection{OBJETIVOS ESPECÍFICOS}

- Discorrer sobre o uso crescente de Ritalina no meio acadêmico.

- Noticiar a indicação médica adequada da Ritalina.

- Listar os possíveis efeitos colaterais no uso da Ritalina.

- Salientar as possíveis conseqüências com o uso da Ritalina sem a prescrição médica.

- Ressaltar a importância da atenção farmacêutica no uso da Ritalina.

\section{METODOLOGIA}

O presente trabalho trata-se de uma revisão bibliográfica, onde analisa o uso indiscriminado e os efeitos colaterais do metilfenidato, conhecido no Brasil como Ritalina. Através da análise qualitativa de estudos já realizados sobre o tema supracitado, foi possível fazer uma revisão de leitura sistemática com o auxílio de sites acadêmicos e artigos científicos que continham em seus títulos os seguintes descritores em ciências da 
saúde: Ritalina, Metilfenidato, uso da Ritalina em acadêmicos, automedicação e o uso racional através da orientação do farmacêutico.

\section{JUSTIFICATIVA}

A importância do presente trabalho está em abordar o uso da Ritalina por pessoas saudáveis, sendo a maioria destes, acadêmicos. É notório que muitos ainda desconhecem a indicação adequada desse fármaco e acabam fazendo uso diário de forma errônea, sendo utilizado para auxiliar na concentração, realização de tarefas acadêmicas e aprimoramento intelectual, o que tornou o país como segundo colocado no uso da Ritalina, onde a cada ano aumenta mais o consumo dessa substância, desconsiderando os malefícios que o mesmo pode causar quando utilizado sem prescrição médica em pessoas saudáveis, sendo possível destacar perturbações mentais, estado de pânico e alucinações.

O profissional farmacêutico tem um papel muito importante na dispensação desse medicamento, é um profissional capacitado e habilitado para praticar atenção farmacêutica com a finalidade de prevenir, identificar e contornar problemas relacionados à farmacoterapia.

\section{DESENVOLVIMENTO}

\section{I. Ritalina}

Ritalina é um psicoestimulante, prescrito para o tratamento de crianças com diagnóstico de TDAH, um estimulante que consumido em doses corretas, pode ajudar no tratamento, como auxílio para o desempenho de tarefas (SILVA et al.,2012). Ao melhorar certas atividades cerebrais, a Ritalina proporciona mais qualidade de vida para crianças e adultos diagnosticados com TDAH.

A Ritalina (metilfenidato) é importante para a regulação da atenção, inibe estímulos que distraem, pensamentos irrelevantes, tira o cansaço, ajuda no aumento de foco e atenção, aumenta a capacidade de ficar acordado por mais tempo e esses efeitos podem ser produzido no organismo sadio (NUNES, et al.; 2020). 
O diagnóstico de TDAH deve ser realizado de acordo com o manual de diagnóstico e estatística dos transtornos mentais e através de uma investigação neurológica minunciosa, para que não haja erro no diagnóstico e posteriormente no tratamento, levando em conta as reações adversas que o fármaco provoca no SNC (BACELAR, 2018). Devido a estes efeitos adversos alguns médicos acabam não aderindo seu uso para qualquer tipo de tratamento. O uso da Ritalina pode ocasionar acatisia, alteração do humor, insônia, sendo necessário destacar que o uso a longo prazo pode ocasionar alucinações e dependência, entre outros. Dessa forma, é extremamente necessário o uso do medicamento com acompanhamento de um médico especialista, uma vez que seu uso de forma inadequada pode levar a um quadro clínico de piora da atenção e cognição, podendo apresentar surtos psicóticos com riscos de cometer suicídio.

Esse fármaco não deve ser usado por crianças menores de seis anos de idade, pois ainda não há comprovação científica quanto a segurança e a eficácia em grupos com essa faixa etária, sendo de suma importância destacar que o mau uso e abuso da Ritalina pode ocasionar sérios problemas psicológicos.

Quando a Ritalina é utilizada corretamente por pessoas que de fato necessitam do fármaco, ocorre o equilíbrio das funções executoras, proporcionando a potencialização da concentração, diminui a perda do foco e da fácil distração, melhora no desempenho escolar, queda da impulsividade, maior atenção em atividades cotidianas, redução da inquietude física e mental, menor ocorrência de sono em momentos imprevisíveis.

\subsection{Mecanismo de ação da Ritalina}

No homem, o sistema nervoso central (SNC) é responsável por receber e processar informações. Essas informações são distribuídas através os neurônios, células pelas quais os impulsos nervosos se propagam até que cheguem à célula alvo. A comunicação entre os neurônios é feita por meio de sinapses, predominantemente químicas, que fazem uso de neurotransmissores - substâncias químicas capazes de causar uma resposta. Os neurotransmissores agem sobre a célula receptora, e em circunstâncias normais cada tipo de informação requer determinados neurotransmissores para transmiti-la ao neurônio seguinte (BACELAR et al., 2018). 
Segundo ITABORAHY (2009) o metilfenidato possui o mesmo mecanismo de ação de substâncias como a cocaína, que é altamente viciante. Esse tipo de substância aumenta a concentração de dopamina, um neurotransmissor associado a satisfação, que possui um efeito de encanto no cérebro. Isso pode estimular o paciente a querer tomar mais do que a dose recomendada, tornando-o dependente.

O metilfenidato age no sistema nervoso central inibindo a recaptação de dopamina neurotransmissor responsável pelo controle motor, e de noradrenalina - neurotransmissor responsável pela excitação física, mental e de bom humor. Dessa forma o fármaco impede que as catecolaminas sejam recapturadas pelas terminações nervosas, assim os neurotransmissores permanecem ativos por mais tempo no espaço sináptico dando ao indivíduo um aumento da concentração, coordenação motora e excitação (BACELAR et al., 2018). É possível visualizar esse mecanismo de ação na figura I.

Figura 1. Mecanismo de ação da Ritalina

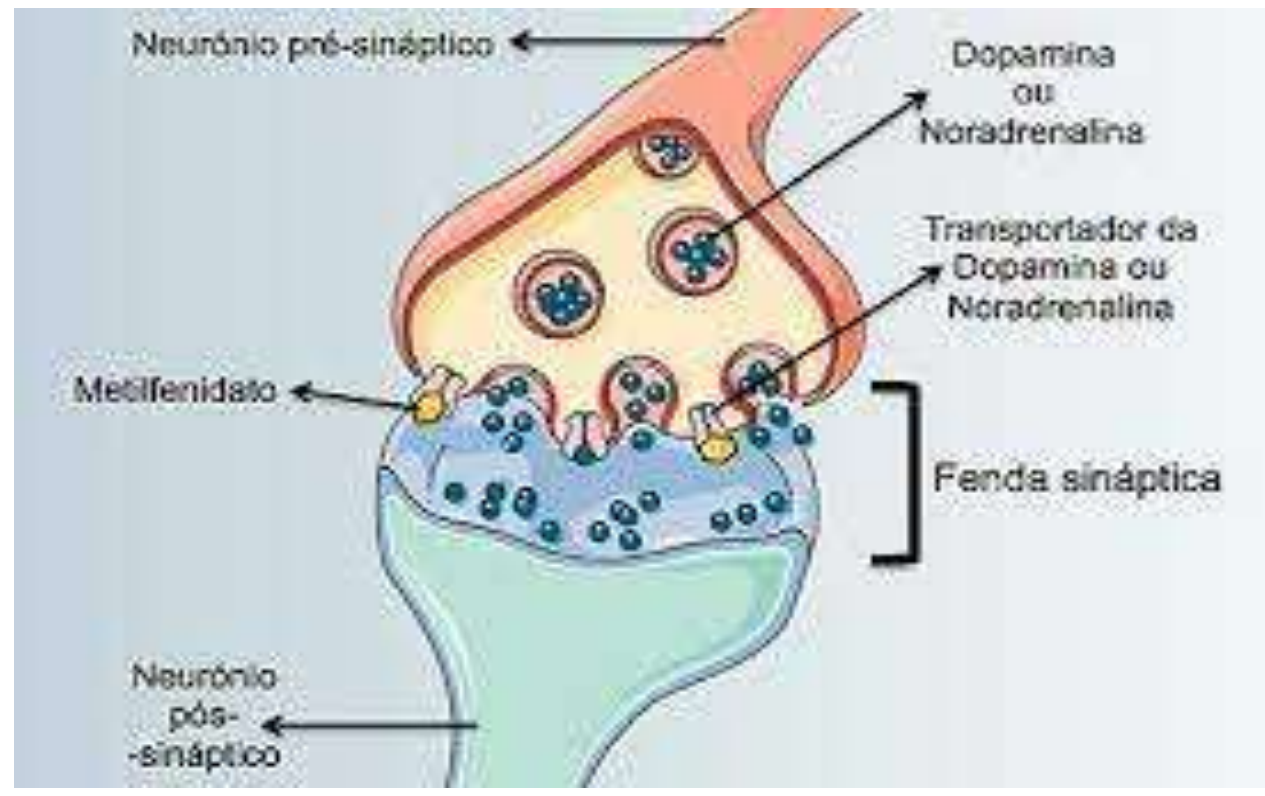

Fonte: Adaptado de MELO et al., 2020

\subsection{EFEITOS COLATERAIS}

Os medicamentos produzem curas, facilitam o convívio dos indivíduos com suas enfermidades, prolongam a vida e retardam o surgimento de complicações referentes às 
doenças. Porém, o uso inadequado dos medicamentos pode aumentar os custos de atenção à saúde ou levar à ocorrência de efeitos colaterais (MELO et al,2020). O uso da ritalina sem orientação médica pode acarretar efeitos colaterais a curto e a longo prazo, além de contar com a possibilidade de causar dependência química. Os efeitos colaterais de curto prazo mais frequentes são: insônia, cefaleia, dores abdominais, redução do apetite, aumento dos batimentos cardíacos, náuseas, alucinações, irritabilidade, dilatação de pupilas, aumento da pressão sanguínea e temperatura corporal. Doses elevadas de Ritalina podem levar a convulsões, espasmos e até mesmo a morte (MELO et al., 2020)

Os efeitos colaterais que podem ocorrer a longo prazo incluem danos pulmonares, renais e hepáticos, perda de peso, confusão mental, cansaço excessivo, danos cerebrais, destruição dos tecidos nasais se o medicamento for inalado, e abcessos se for injetado. Existem três efeitos colaterais de longo prazo de maior importância da Ritalina: a dependência, os efeitos cardiovasculares e a possível redução da estatura. (MELO et al, 2020).

A interrupção brusca do metilfenidato pode ocasionar a síndrome de abstinência, além da insônia, sonolência, piora na atenção e na cognição, surtos psicóticos, alucinações e em casos mais graves suicídio.(NUNES, 2020)

\subsection{O uso da Ritalina por acadêmicos}

Entre os acadêmicos a popularização da Ritalina tem aumentado a cada dia, onde se tornou muito comum ouvir entre os estudantes que fez uso da Ritalina para passar a noite estudando, essa prática que popularmente é conhecida como (doping mental). O problema dessa prática é que muitos desses usuários não conhecem os efeitos colaterais que esse medicamento proporciona.

Após a leitura de várias pesquisas já realizadas sobre o tema supracitado, fica em evidência que os estudantes não encontram dificuldades na hora de adquirir o medicamento, mesmo se tratando de um medicamento que exige a apresentação da receita médica no ato da compra, pois o mesmo é facilmente encontrado em sites ilegais na internet, o que aumenta o risco do uso indiscriminado da droga, outra estratégia que alguns estudantes usam é simulando os sintomas de TDAH na tentativa de conseguir um 
diagnóstico positivo mesmo sabendo que se trata de um diagnóstico equivocado, porém dessa forma é possível ter a garantia de conseguir adquirir o medicamento de forma legal.

\subsection{A importância da atenção farmacêutica}

Ao analisar as funções do farmacêutico no sistema de atenção a saú de, a Organização Mundial de Saúde - OMS estende o benefício da atenção farmacêutica para toda comunidade reconhecendo a relevância da participação do farmacêutico junto com a equipe de saúde na prevenção de doenças e promoção da saúde. Na ótica da OMS a atenção farmacêutica é um conceito de prática profissional na qual o paciente é o principal benefici ário das ações do farmacêutico. A atenção farmacêutica é o compêndio das atitudes, os comportamentos, os compromissos, as inquietudes, os valores éticos, as funções, os conhecimentos, as responsabilidades e as habilidades do farmacêutico na prestação da farmacoterapia com o objetivo de obter resultados terapêuticos definidos na saúde e na qualidade de vida do paciente" (REIS, 2003).

Segundo a Política Nacional de Medicamentos o uso racional de medicamentos é um procedimento que compreende a prescrição adequada, a disponibilidade conveniente a preços acessíveis, a dispensação em circunstâncias adequadas, seu consumo em doses indicadas, intervalos exatos e no período de tempo indicado.

A atenção farmacêutica contribui para o uso racional de medicamentos, buscando garantir a segurança e a efetividade no processo de utilização de medicamentos, auxiliando na obtenção de melhores resultados durante a farmacoterapia.

A Ritalina se trata de um fármaco da Classe $A_{3}$ (Psicotrópicos) que para sua obtenção há a necessidade de uma receita de controle especial, e, sua dispensação deve ser realizada através do profissional farmacêutico, onde o mesmo tem a responsabilidade de esclarecer quaisquer dúvidas sobre como utilizar o medicamento de forma adequada.

É importante ressaltar que a aquisição da Ritalina pela internet sem a solicitação da apresentação de receita médica é considerada crime de acordo com o Art. 33 da lei II.343/2006, sob a pena de reclusão de 5 a is anos e multa. 


\section{DISCUSSÃO}

A Ritalina é indicada a pessoas que sofrem de Transtorno de Déficit de Atenção (TDAH) em doses que cabem exclusivamente a um médico receitar.

O Metilfenidato é hoje o psicoestimulante mais consumido no mundo, devido a popularização do TDAH e por suas propriedades psicoestimulantes, com isso estudantes utilizam o metilfenidato de maneira incorreta a fim de ter um maior rendimento intelectual.

A grande preocupação do uso do metilfenidato é a utilização do fármaco por pessoas saudáveis, que ignoram as conseqüências que esse medicamento pode acometer a saúde, principalmente quando usado a longo prazo, podendo desenvolver patologias crônicas.

\section{CONSIDERAÇÕES FINAIS}

Este trabalho teve por objetivo esclarecer os riscos da utilização da Ritalina por acadêmicos, uma vez que o número de estudantes que adotam esse método para atingir seus objetivos cresce a cada dia, alertando sobre os efeitos colaterais a curto e a longo prazo além da dependência que pode ocorrer pelo uso abusivo dessa medicação.

O profissional farmacêutico pode auxiliar no uso racional de medicamentos sempre atentando sobre os possíveis riscos que podem surgir com a farmacoterapia.

Portanto, fica em evidência que o público universitário tem a necessidade de uma conscientização sobre o uso da Ritalina, apontando para os prejuízos e os riscos que podem ocorrer à saúde devido ao uso desse medicamento de forma indevida

\section{REFERÊNCIAS}

BACELAR, Ana Beatriz. Ritalina uma droga que ameaça a inteligência. Revista de Medicina e Saúde de Brasília 20I8; 7 (I): 99-II2.

BOLETIM INFORMATIVO DO CIM - RS. Farmacovigilência: Reações adversas a Medicamentos. Disponível em: 〈http://www.ufrgs.br/boletimcimrs/RAM\%202008.pdf.> Acesso em: 15/09/2021. 
Conselho Federal de Farmácia, Resolução no 357 de 20 de abril de 2021. Aprova o regulamento técnico das boas práticas de Farmacia. Disponível em: 〈http://www.cff.org.br/userfiles/file/resoluções/357.pdf.> Acesso em I2/o9/202I.

CRUZ, Tarcisio C. S. C et al., Uso Não-Prescrito de Matilfenidato entre Estudantes de Medicina da Universidade Federal da Bahia. Gaz. Med. Bahia 2011;81:I(Jan - Jun)

ESHER, Angela; COUTINHO, Thiago. Uso Racional de Medicamentos, Farmaceticalização e usos do Metilfenidato. Ciências \& Saúde Coletiva, 22(8):2571-2580, 2017.

FARDIN, Carlos Eduardo; PILOTO, Juliana Antunes da Rocha. Uso indiscriminado do metilfenidato para o aperfeiçoamento cognitivo em indivíduos saudáveis. Revista Uningá Review. Vol. $23-\mathrm{n}^{\circ}$, 2015 .

FILHO, João Pereira et al., Medicação de alto risco: reflexão da Ritalina. Revista Eletrônica Acervo Saúde / ISSN 2178-2091, Vol. 12-2020.

ITABORAHY, Cláudia. A Ritalina no Brasil: uma década de produção, divulgação e consumo. Universidade do estado do Rio de Janeiro. Rio de Jnaeiro, 2009.

JR, Walter Camargos; NICOLATO, Rodrigo. Características das prescrições no transtorno de déficit de atenção/hiperatividade. Jornal Brasileiro de Psiquiatria 58 (3), I95199, 2009.

MADRUGA, Célia Maria Dias; SOUZA, Euripides Sebastião Mendonça. Manual de Orientações básicas para prescrição médica. Disponível em: 〈http://portal.cfm.org.br/imagens/stories/biblioteca/cartilhaprescrimed2o12.pdf.〉 Acesso em: 2I/o8/202I. 
MElO, R. S.; FELICIONI, F.; AFONSO, A. C. B.; SOUZA, N. B. Ritalina: Consequências pelo uso abusivo e orientações de uso. Revista científica Online ISSN i980$6957-\mathrm{VI2}, \mathrm{nI}, 2020$.

NUNES, Solange da Silva. O uso da Ritalina por acadêmicos: Desenvolvimento Acadêmico sob o efeito da Ritalina. Ariquemes: FAEMA, 2020.

PIRES, M. S.; DIAS, A. P. ; PINTO, D. C. L.; GONÇALVES, P. G.; SEGHETO, W. O Uso de Substâncias Psicoestimulantes sem Prescrição Médica por Estudantes Universitários. Revista Científiva Fagoc Saúde - Vol III - 2018.

RITALINA: Cloridrato de Metilfenidato. Novartis Biociência S.A. Farm. Resp.: Flávia Regina Pegorer - CRF - SP i8.150, 2015. Bula de Remédio.

ROSA, Amanda Freitas et al., Uso de Matilfenidato (Ritalina) por estudantes de Medicina de um Centro Universotário de Porto Velho. Revista Eletrônica Acervo Saúde/ISSN 21782091, Vol I3.

SILVA et al., A Explosão do consumo de Ritalina. Revista de Psicologia da UNESP I (2), 2012.

SOUZA, A. C. P.; LUZIO, C. A.; SANTOS, K. Y. P.; YASUI, S.; DIONÍSIO, G, H. Descrição e Utilização do Metilfenidato. Revista Científica Eletrônica de Ciências Aplicadas da FAIT. N. I. Maio, 2021.

VAZ, B. P.; BOTELHO, F. L. R.; LOURENÇO, J. S.; ESPER, M. N. D.; TORRES, V. M. F.; OLIVEIRA, R. R. Uso de Metilfenidato por estudantes universitários. Revista Educação em Saúde: v8, suplemento I, 2020. 